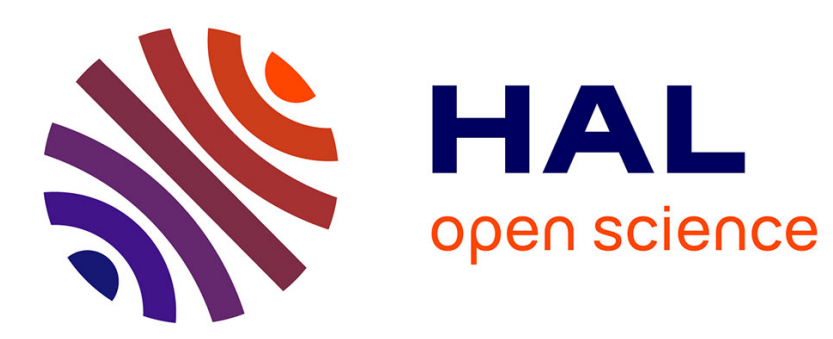

\title{
Actions of groups of finite Morley rank on small abelian groups
}

Adrien Deloro

\section{To cite this version:}

Adrien Deloro. Actions of groups of finite Morley rank on small abelian groups. Bulletin of Symbolic Logic, 2009, 15 (1), pp.70-90. 10.2178/bsl/1231081770 . hal-01303300

\section{HAL Id: hal-01303300 \\ https://hal.science/hal-01303300}

Submitted on 17 Apr 2016

HAL is a multi-disciplinary open access archive for the deposit and dissemination of scientific research documents, whether they are published or not. The documents may come from teaching and research institutions in France or abroad, or from public or private research centers.
L'archive ouverte pluridisciplinaire HAL, est destinée au dépôt et à la diffusion de documents scientifiques de niveau recherche, publiés ou non, émanant des établissements d'enseignement et de recherche français ou étrangers, des laboratoires publics ou privés. 


\title{
ACTIONS OF GROUPS OF FINITE MORLEY RANK ON SMALL ABELIAN GROUPS
}

\author{
ADRIEN DELORO
}

\begin{abstract}
We classify actions of groups of finite Morley rank on abelian groups of Morley rank 2: there are essentially two, namely the natural actions of $\operatorname{SL}(V)$ and $\operatorname{GL}(V)$ with $V$ a vector space of dimension 2. We also prove an identification theorem for the natural module of $\mathrm{SL}_{2}$ in the finite Morley rank category.
\end{abstract}

§1. Introduction. The introduction has three moments. $\S 1.1$ recasts general ideas on groups of finite Morley rank. The results of the present article are exposed in $\S 1.2$. We comment on the main proof and its tools in $\S 1.3$.

1.1. Generalities on groups of finite Morley rank. Groups of finite Morley rank made their first appearance in strictly model-theoretic contexts as groups whose first-order theory is $\aleph_{1}$-categorical. Quickly Cherlin and Zilber both conjectured that simple infinite groups of finite Morley rank should be algebraic groups over algebraically closed fields-following the informal idea that only the usual geometry can provide objects which are so well-behaved, logically speaking. This "algebraicity conjecture" is not too surprising if one bears in mind that the Morley rank may be viewed as an abstract model-theoretic generalization of the Zariski dimension.

Actually it has even been proved by Borovik and Poizat that for any group $G$ in a first-order language $\mathcal{L}$ (containing of course the language of groups) whose collection of definable sets is equipped with a notion of dimension satisfying a couple of natural axioms, the structure $(G, \mathcal{L})$ has finite Morley rank. Let us be more precise. By definable we mean first-order interpretable (understand: with parameters and definable quotients); the axioms are obvious properties of the Zariski dimension on the collection of constructible sets of an algebraic group.

As a consequence of Borovik and Poizat's result, no solid model-theoretic background is required to read papers on groups of finite Morley rank, or at least to read this one: we merely deal with an abstract notion of dimension (called rank) on definable sets.

Received July 26, 2008.

The author is a recipient of the Sacks Prize. 
From the discussion above it seems that groups of finite Morley rank should be a natural model-theoretic generalization of algebraic groups. Many tools from algebraic group theory, such as the notion of a connected component for definable subgroups, generation by indecomposable subsets containing the identity, interpretability of a field, do translate indeed into finite Morley rank (abstract) analogs. For these points and every time the reader needs further details, we refer to [13]; [5] is another adequate reference.

On the other hand, to give an idea of the difficulty of the situation, one does not know how to classify groups of Morley rank 3. This is the most depressing part of the more general thirty-year open question of bad groups: is there a simple group of finite Morley rank in which all definable, solvable, connected subgroups are nilpotent?

In spite of such potentially non-algebraic configurations the conjecture has met a partial positive answer under extra assumptions on the structure of the Sylow 2-subgroup ([2], the core result of which we do not use). This way of handling groups of finite Morley rank, inspired by the Borovik program, is where groups of finite Morley rank meet finite group theory, and however essential the connection may be, we shall try not to focus on this aspect here. It nonetheless explains why people in groups of finite Morley rank are raving for involutions.

1.2. The results. Regardless of the huge body of work the Cherlin-Zilber algebraicity conjecture has stirred, little is known about group actions of finite Morley rank, i.e., a group, a set, and a group action on the set, all three objects being definable in a structure of finite Morley rank. [12] (whose results we shall not use) laid some foundations. Recently more material has been exposed in [4] (which we take for our reference on permutation groups of finite Morley rank), and in particular the problem we shall consider below.

Quite interestingly, natural questions in the algebraic category such as determining the degree of generic transitivity of an algebraic group action have been solved only recently [15], and only in the characteristic 0 case. [4] suggests that perhaps logicians should try to tackle such questions by their own means. We address a problem of this kind.

In [4] Borovik and Cherlin posed 18 problems including the following.

Problem 15 of [4]. Let $G$ be a connected group of finite Morley rank acting faithfully and definably on an abelian group $V$ of Morley rank 2 . Then either $G$ is solvable or $V$ has a structure of a 2-dimensional vector space over an algebraically closed field $F$ and $G$ is one of the groups $\operatorname{SL}_{2}(F)$ and $\mathrm{GL}_{2}(F)$ in their natural representations.

This question appears as a very first step towards a more ambitious one, namely the classification of generically $n$-transitive actions $(G, V)$, where $G$ is a permutation group of the abelian group $V$ (all objects being definable in a finite Morley rank structure). We can but refer the reader to $[4, \S 6]$ for 
further details; it should however be clear that we are dealing with the analog of a natural algebraic question in a more general, logic-flavored setting.

As for Problem 15, we provide a positive answer.

THeOREM A. Let $G$ be a connected, non-solvable group of finite Morley rank acting definably and faithfully on a connected abelian group $V$ of Morley rank 2. Then there is an algebraically closed field $\mathbb{K}$ of Morley rank 1 such that $V \simeq \mathbb{K}^{2}$, and $G$ is isomorphic to $\mathrm{GL}_{2}(\mathbb{K})$ or $\mathrm{SL}_{2}(\mathbb{K})$ in its natural action.

Investigating questions related to Theorem A, we could also show the following on $\mathrm{SL}_{2}$-modules.

THEOREM B. Let $\mathbb{K}$ be a field of Morley rank $k$. Let $G$ be a group of finite Morley rank isomorphic to $\mathrm{SL}_{2}(\mathbb{K})$ acting definably and non-trivially on a connected abelian group $V$ of rank $\leq 2 k$. Then $V$ is a natural module for $G$.

Both results are independent. Theorem $\mathrm{A}$ is the main result of the present article; Theorem $\mathrm{B}$ is proved in $\S 5$.

1.3. On the proof of Theorem A. If $G$ as in Theorem A was known to be algebraic, a proof would be rather easy using the classification of algebraic groups. Of course we do not make such an assumption, and we have to deal with Theorem A in a more direct, logic-oriented fashion. So perhaps the proof we shall expose is interesting regardless of the result.

Let us give a rough account of the landscape by reviewing some of the required notions (technical prerequisites are exposed in $\S 2$ ).

- Many results on linear actions are consequences of Zilber's celebrated field theorem (Fact 2.1 below), a general method which produces an algebraically closed field in a solvable, non-nilpotent connected group of finite Morley rank.

- The Hrushovski analysis [13, Théorème 3.27] is the classification of groups of finite Morley rank acting faithfully on strongly minimal setsthe only non-solvable one is $\mathrm{PSL}_{2}$ (on a coset space).

- As for unipotence, two families of notions are in use. The finite characteristic case is a fairly natural modelization of unipotent subgroups of an algebraic group over a field of prime characteristic: a p-unipotent subgroup of a group of finite Morley rank is merely a definable, connected, nilpotent subgroup of (finite) exponent a power of $p$. (Quite remarkably when $p \neq 2$, "nilpotent" is essential in the definition.)

- Unipotence in null characteristic appeared in [6] (see also [7]) and does not enjoy so natural a definition. Though this tool is in general complex to manipulate we shall pretend it is not. There are two reasons to do so. First, in our setting, Burdges' reduced rank (or "unipotence degree") will take only 1 as a non-trivial value. Second, null charateristic unipotence might be avoided by providing another proof in case $V$ is torsion-free. This alternative proof of Theorem A (to be found in $\S 4$ ) 
is shorter but relies on the analysis of bad groups, and does not generalize to the case where $V$ is torsion. These are the reasons that made us prefer a more technical, but more uniform, argument.

- Jaligot Lemmas deal with rigidities in group-theoretically "small" groups of finite Morley rank. They mimic the fact that in $\mathrm{PSL}_{2}$ or $\mathrm{SL}_{2}$, unipotent subgroups belong to a unique Borel subgroup - a Borel subgroup of a group of finite Morley rank being a definable, connected, solvable subgroup maximal with respect to these properties. The first version of a Jaligot Lemma in null characteristic goes back to [10]. For the latest version and the notion of smallness one might want to see [11].

- Good tori appeared in [6] (see also [7]) and are the abstract analog of algebraic tori. When opposed to Burdges' unipotent subgroups, good tori are our modelization of semi-simplicity.

Some of these ingredients are collected in $\S 2$; the proof of Theorem A is to be found in $\S 3$. Recall that a shorter proof in a special case is available in $\S 4$.

Acknowledgements. Many, many thanks to Alexandre Borovik and Gregory Cherlin for very helpful conversations. Extra thanks to Gregory Cherlin for discussions on Theorem B.

§2. The tools. We collect here some raw meat. The proof of Theorem A takes place in $\S 3$.

2.1. Linear actions. Let us first recast some finite Morley rank theorems around $\mathrm{GL}_{n}$. Remember that given a group $H$, an infinite definable $H$ invariant group $A$ is $H$-minimal if it contains no proper infinite definable $H$-invariant subgroup.

FACT 2.1 (Zilber Field Theorem, [13, Théorème 3.7]). Let $A$ be a definable abelian group with an infinite abelian group of automorphisms $M$ definable inside a structure of finite Morley rank. If $A$ is $M$-minimal, then there is an infinite definable field $\mathbb{K}$ and a definable $\mathbb{K}$-vector space structure of dimension 1 on $A$ such that $M$ acts $\mathbb{K}$-linearly, i.e., $A \simeq \mathbb{K}_{+}$and $M \hookrightarrow \mathbb{K}^{\times}$ definably.

Here is the consequence we shall use when retrieving the pair $\left(\mathrm{GL}_{2}(\mathbb{K}), \mathbb{K}^{2}\right)$.

FACT 2.2. [13, Théorème 3.9] Let $G$ be a connected group of finite Morley rank acting definably and faithfully on a definable, abelian group A. Assume that $G$ has a definable, connected, normal subgroup $H \unlhd G$ which has an abelian, infinite, normal subgroup $M \unlhd H$, and such that $A$ is $H$-minimal. Then there is a definable field $\mathbb{K}$ and a definable finite-dimensional $\mathbb{K}$-vector space structure on $A$ such that $G$ embeds into $\mathrm{GL}(A)$. Moreover, $M$ acts on $A$ by scalar multiplication.

Another corollary we shall use of Fact 2.1 is the following abstract analog of the Lie-Kolchin-Malcev Theorem. 
FACT 2.3 (Malcev's Theorem [13, Théorème 3.18]). Let $G$ be a connected, solvable group of finite Morley rank acting definably and faithfully on a definable, abelian group $A$. If a definable subgroup $B \leq A$ is $G$-or $G^{\prime}$-minimal, then $B$ is centralized by $G^{\prime}$.

2.2. Around $\mathrm{PSL}_{2}$. Fact 2.2 will enable us to recognize $\left(\mathrm{GL}_{2}(\mathbb{K}), \mathbb{K}^{2}\right)$. On the other hand, the identification of the action $(G, V)$ with $\left(\mathrm{SL}_{2}(\mathbb{K}), \mathbb{K}^{2}\right)$ will be a little harder. We have found no better way than first identifying $G$ with $\mathrm{SL}_{2}(\mathbb{K})$, then claiming that it acts "naturally". The second step is described in $\S 2.3$.

For the first step we will make use of the Hrushovski analysis, which identifies a non-solvable group acting faithfully on a strongly minimal set (in a finite Morley rank context) with $\mathrm{PSL}_{2}(\mathbb{K})$ for some algebraically closed field $\mathbb{K}$. So we shall first find a quotient of $G$ isomorphic to $\mathrm{PSL}_{2}$, and then lift the information.

Here is a consequence of the Hrushovski analysis not entirely explicit in [13, Corollaire 3.28].

FACT 2.4. Let $G$ be a non-solvable group of finite Morley rank having a definable subgroup $H$ of corank 1 such that $\bigcap_{g \in G} H^{g}=1$. Then $G$ has Morley rank 3 and is isomorphic to $\mathrm{PSL}_{2}(\mathbb{K})$, where $\mathbb{K}$ is a definable field of Morley rank 1.

Proof. To see this, it suffices to note that $G$ acts definably, transitively and faithfully on the definable coset space $G / H$ which is strongly minimal. So the Hrushovski analysis [13, Théorème 3.27] yields the conclusions.

At some point of the proof of Theorem A we shall thus have a quotient of $G$ isomorphic to $\mathrm{PSL}_{2}$. What if this is $G$ itself? We shall therefore need to rule out from the picture $\mathrm{PSL}_{2}(\mathbb{K})$ in characteristic not 2. (Of course if $\mathbb{K}$ has characteristic 2 , then $\operatorname{PSL}_{2}(\mathbb{K})=\mathrm{SL}_{2}(\mathbb{K})$ will certainly remain.) This is done by the following remark.

FACT 2.5. Let $\mathbb{K}$ be a field of finite Morley rank of characteristic $\neq 2$. Then there is no definable, non-trivial action of $\mathrm{PSL}_{2}(\mathbb{K})$ on an abelian group of rank 2.

Proof. Let $\operatorname{PSL}_{2}(\mathbb{K})$ act on such an abelian group $V$, and let us prove a contradiction. The action is obviously faithful. We first notice that $V$ is not an elementary abelian 2-group, as otherwise letting $\tau \leq \mathrm{PSL}_{2}(\mathbb{K})$ be a 2-torus, we find that $V \rtimes \tau=V * \tau$, whence $\tau \leq C_{G}(V)=1$. This means that $\mathbb{K}$ has characteristic 2, against our assumption.

Hence $V$ does not have characteristic 2. Let $i \in \mathrm{PSL}_{2}(\mathbb{K})$ and $w \in$ $C(i) \backslash\{i\}$ be two commuting involutions. As $i$ is not central in $\mathrm{PSL}_{2}(\mathbb{K})$, we find a decomposition $V=C_{V}(i) \oplus[V, i]$ and both terms are non-trivial. Notice that $w$ normalizes them; each is either centralized or inverted by $w$.

If $w$ centralizes $C_{V}(i)$, then $w \neq 1$ cannot centralize $[V, i]$ too, and must therefore invert it. It follows that $i$ and $w$ have the same action on $V$, whence 
$w=i$, a contradiction. Now if $w$ does not centralize $C_{V}(i)$, then it inverts it. As $w$ is not central in $\operatorname{PSL}_{2}(\mathbb{K})$, it cannot invert $[V, i]$ too, so it must centralize it. It follows that $i w$ inverts $V$, so $i w$ must be central in $\operatorname{PSL}_{2}(\mathbb{K})$, a contradiction again.

2.3. Around $\mathrm{SL}_{2}$. We will still have to argue that when making $G$ as in Theorem $\mathrm{A}$ isomorphic to $\mathrm{SL}_{2}(\mathbb{K})$ via an à la Hrushovski analysis as in $\S 2.2$, we actually identify the pair $(G, V)$. In other words, we have to determine small representations of $\mathrm{SL}_{2}$. This is possible thanks to the following, originally due to Timmesfeld.

FACT 2.6. [2, Fact II.5.28] Let $\mathbb{K}$ be a field and let $V$ be an $\mathrm{SL}_{2}(\mathbb{K})$-module. Suppose the following:

1. $C_{V}(G)=0$ and $[G, V]=V$,

2. $V$ is faithful and $[U, U, V]=1$, where $U$ is a maximal unipotent subgroup of $G$.

Then for some field action on $\left\langle v^{G}\right\rangle$, the vector space $\left\langle v^{G}\right\rangle$ is a natural module for each $v \in C_{V}(U)^{\#}$.

(Faithfulness is part of Timmesfeld's definition of quadratic, which is the condition on commutators above. This is implicit in our source [2] too.) Here is the consequence we shall use.

FACT 2.7. Let $\mathbb{K}$ be a field of Morley rank 1 and let $G \simeq \mathrm{SL}_{2}(\mathbb{K})$ act definably and non-trivially on a connected abelian group $V$ of Morley rank 2. Then $V$ is a natural module for $G$.

Proof. The action is clearly faithful by Fact 2.5. We apply Fact 2.6. Let $W=C_{V}(G)$ and $\bar{V}=V / W$. Cleary $W$ is finite, so $\bar{V}$ has rank 2 and $C_{\bar{V}}(G)=0$; furthermore $[G, \bar{V}]=\bar{V}$. Let $U$ be a maximal unipotent subgroup of $G$, and $B=N_{G}(U) . \bar{V}$ is not $B$-minimal, as otherwise $B^{\prime}=1$ by Fact 2.3 and faithfulness. So there is an infinite $\bar{V}_{1}<\bar{V}$ which is $B$-invariant. Now by Fact $2.3, U$ centralizes both $\bar{V}_{1}$ and $\bar{V} / \bar{V}_{1}$, so $[U, U, \bar{V}]=1$. By Fact 2.6, $\bar{V}$ is a natural module for $G$.

If $W=0$ we are of course done. So assume $W \neq 0$, we shall find a contradiction. As $V$ is $G$-minimal, $V$ has exponent $p$. Furthermore one sees easily that char $\mathbb{K}=p$.

If $x \in V \backslash W$, then its image $\bar{x} \in \bar{V}$ is such that $C_{G}(\bar{x})$ is a maximal unipotent subgroup of $G$. In particular it is connected. Now $\left[C_{G}(\bar{x}), x\right]$ is a connected subgroup of $W$ which is finite. As a conclusion, $C_{G}(x)=$ $C_{G}(\bar{x})$. Hence all elements of $V \backslash W$ have a generic orbit in $V$, so there is a unique generic orbit $O \subseteq V$. It follows that there is a translate $v+W$ of $W$ that is contained in $O$, as otherwise $V \backslash O$ would be generic too, against connectedness. Fix $w \in W^{\#}$; we find two elements of $V, x$ and $y=x+w$ which are $G$-conjugate: $y=x^{g}$. Applying $g$ again and again, we get $g^{p} \in C(x)$. But $C(x)$ is a maximal unipotent subgroup and $\mathbb{K}$ has 
characteristic $p$, so $C(x)$ has no extension of degree $p$; if follows $g \in C(x)$ and we find $y=x$, and $w=0$. This contradiction proves $W=0$.

Therefore $V=\bar{V}$ is a natural module for $G$.

By the way, what happens in higher rank? using the same characterization (Fact 2.6), Altinel, Borovik, and Cherlin proved:

FACT 2.8. [2, Lemma II.5.31] Let $G$ be a group of finite Morley rank that is isomorphic to $\mathrm{SL}_{2}(\mathbb{K})$ as an abstract group with $\mathbb{K}$ an algebraically closed field of characteristic 2 . Let $V$ be a connected, elementary abelian 2-group on which $G$ acts definably and faithfully. Let $f=\mathrm{rk} \mathbb{K}$, and suppose $\mathrm{rk} V=2 f$. Then $V$ is a natural module for $G$.

(In the original, connectedness of $V$ was however omitted, or implicit.)

As a dessert we shall prove the following in $\S 5$.

THEOREM B. Let $\mathbb{K}$ be a field of Morley rank $k$. Let $G$ be a group of finite Morley rank isomorphic to $\mathrm{SL}_{2}(\mathbb{K})$ acting definably and non-trivially on a connected abelian group $V$ of rank $\leq 2 k$. Then $V$ is a natural module for $G$.

Theorem B is an analog of Fact 2.8 in characteristic not 2. Note however that Fact 2.7 is sufficient to establish Theorem A.

2.4. Further remarks on $\mathrm{SL}_{2}$. The following result will be the basis of our analysis. It will yield solvability of centralizers (Claim 3.5 below), a good starting point when one wishes to talk about Borel subgroups. We sketch a couple of proofs, hoping one of them will seem of interest to the reader.

Lemma 2.9. Let $G$ be a connected group of finite Morley rank acting definably and faithfully on a connected abelian group $V$ of Morley rank 2. Let $v_{0} \in V^{\#}$. Then $C_{G}^{\circ}\left(v_{0}\right)$ is not isomorphic to $\mathrm{SL}_{2}$ nor to $\mathrm{GL}_{2}$.

Proof. We may assume that there is a subgroup $H \leq C_{G}^{\circ}\left(v_{0}\right)$ which is isomorphic to $\mathrm{SL}_{2}$. Clearly $H$ acts non-trivially on $V$. By Fact 2.7 , we get that $H$ acts on $V$ as $\mathrm{SL}_{2}$. In particular $H \leq C_{G}^{\circ}\left(v_{0}\right)$ is a contradiction.

The proof we just gave relies on an identification result of the natural $\mathrm{SL}_{2}$-module. As we have said after Fact 2.8, we still miss a more general tool when the characteristic differs from 2 and the rank is greater than 2. So the reader might enjoy the following argument, which spares us the concourse of the natural module.

Proof. Assume $H$ is isomorphic to $\mathrm{SL}_{2}(\mathbb{K})$, where $\mathbb{K}$ has characteristic not 2, and $V$ has no involutions. Pick the central involution $i \in Z(H)$. As $V$ is $2^{\perp}$, we may write the decomposition $V=C_{V}(i) \oplus[V, i]=C_{V}^{\circ}(i) \oplus[V, i]$ by connectedness of $V$. Notice that $C_{G}^{\circ}(i)$ normalizes each term; a quick computation shows that if both $C_{V}(i)$ and $[V, i]$ have rank 1, then $C_{G}^{\circ}(i) \geq H$ is solvable, which is absurd. As $[V, i] \neq 1$, it follows that $C_{V}(i)=1$ and $V=[V, i]$, that is $i$ inverts $V$. This contradicts $i \in C_{G}^{\circ}\left(v_{0}\right)$.

The reader may very well not be pleased at all with the latter argument either. In this case we proceed as follows, regardless of the characteristic. 
Alternate Proof of Lemma 2.9. Fix an algebraic torus $T \leq H$ and $j$ inverting $T$ (the order of $j$ depends on the characteristic). Let $B \leq H$ be a Borel subgroup containing $T$, so that $B^{j}$ is the other such Borel subgroup.

We claim that there is a unique $B$-minimal subgroup $V_{1}<V$. Indeed, if $V$ itself is $B$-minimal, then by Fact $2.3 B^{\prime}=1$, which is absurd. So $V_{1}$ does exist, and $B^{\prime}$ centralizes $V_{1}$. If there is another $B$-minimal subgroup, then $B^{\prime}=1$ again. (Notice that we have made crucial use of $\mathrm{rk} V=2$ at this point.) It is clear that $B=N_{H}^{\circ}\left(V_{1}\right)$, so $V_{2}:=V_{1}^{j} \neq V_{1}$.

We now claim that $T$ acts freely on $V_{1}^{\#}$ and $V_{2}^{\#}$. Let $t \in C_{T}\left(V_{1}\right)$; then $t$ centralizes $V_{1}$ and $t^{j}$ centralizes $V_{1}^{j}$, so $t$ centralizes $V_{1}+V_{1}^{j}=V$, whence $t=1$. Hence $C_{T}\left(V_{1}\right)=1$, and by the Zilber field theorem, $T$ embeds into the multiplicative group of a field whose additive group is isomorphic to $V_{1}$. The action is thus free on $V_{1}^{\#}$. The same applies to $V_{2}^{\#}$. This proves our claim.

We claim further that $C_{T}\left(V / V_{1}, V / V_{2}\right)=1$. Let $t \in C_{T}\left(V / V_{1}, V / V_{2}\right)$. Then fixing $v \in V$, there are $v_{1} \in V_{1}$ and $v_{2} \in V_{2}$ such that $v^{t}=v+v_{1}=$ $v+v_{2}$, so $v_{1}=v_{2} \in V_{1} \cap V_{2}$. As this intersection of distinct groups of rank 1 is finite, it follows by connectedness that $[V, t]$ is trivial, and $t=1$.

Hence we can assume that $K:=C_{T}\left(V / V_{1}\right)<T$. In particular, $T / K$ acts freely on $\left(V / V_{1}\right)^{\#}$. Recall that we had started with an arbitrary $v_{0} \in V^{\#}$, and $T \leq H \leq C_{G}^{\circ}\left(v_{0}\right)$. Then $v_{0} \in V_{1}$. As $T \leq C_{G}^{\circ}\left(v_{0}\right)$ acts freely on $V_{1}^{\#}$, we find $v_{0}=0$, a contradiction.

Such ideas relate to Theorem B, proved in $\S 5$, which the reader may wish to enjoy now.

2.5. Unipotence and semi-simplicity. As mentioned in the introduction, our notions of "unipotent-like" and "semi-simple-like" subgroups of a group of finite Morley rank are not as obvious as their algebraic origins.

DeFinition 2.10. A p-unipotent subgroup of a group of finite Morley rank is a definable, connected, nilpotent, p-subgroup of bounded exponent.

In the solvable case, there is a $p$-unipotent radical.

FACT 2.11. [1, Fact 2.36] Let $H$ be a solvable, connected group of finite Morley rank. Then there is a unique maximal p-unipotent subgroup of $H$, denoted $U_{p}(H)$.

Stated as fuzzily as here the following will look more like a slogan than a fact; this is the price to pay if one wishes to avoid Burdges' rather technical definitions.

FACT $2.12([6, \S 2.1]$, see also [7, Chapter II]). There exists a family of notions of unipotence in null characteristic. They are denoted $(\infty, d)$, where $d$ is an integer (equal to the Morley rank of certain abelian torsion-free sections).

For $d_{\infty}(H)$ maximal such that the solvable group $H$ of finite Morley rank contains non-trivial $(\infty, d)$-unipotent subgroups, there is a corresponding $\left(\infty, d_{\infty}(H)\right)$-unipotent radical, denoted $U_{\left(\infty, d_{\infty}(H)\right)}(H)$. 
For convenience, one then lets $\tilde{p}$ denote either a prime characteristic writ-

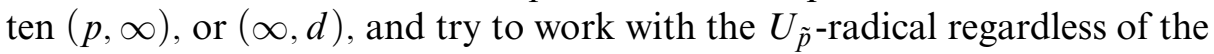
nature of $\tilde{p}$. Such a $\tilde{p}$ is called a unipotence parameter. It is said to be trivial only when of the form $(\infty, 0)$.

Let $H$ be a solvable group of finite Morley rank. When $\tilde{p}$ is of the form $(p, \infty)$ and $U_{\tilde{p}}(H) \neq 1$, or $\tilde{p}=(\infty, d)$ with $d$ maximal such that there are non-trivial $(\infty, d)$-unipotent subgroups of $H$ (i.e., when $d=d_{\infty}(H)$ with the notation of Fact 2.12), one says that $\tilde{p}$ is a maximal unipotence parameter for $H$.

The main issues in characteristic 0 are that maximality is relative (to $H$ ), and unless $d=d_{\infty}(H)$ we might not have something worth calling a $\tilde{p}$ unipotent radical. Fortunately in the present article no subtleties will arise from this.

The reasons why we do not wish to emphasize on Burdges' unipotence notions are that they can be in general fairly tricky to handle, and we shall need only the most naive version, if any version at all. So the inexperienced reader may attack Fact 3.13 below with the finite characteristic intuition; he must however bear in mind that in other articles, the situation is not as friendly. He will find comfort in Section 4, where a short proof of Theorem A is provided in characteristic 0, which spares the use of Burdges' theory. For a full account of unipotence parameters and Jaligot lemmas, we refer to [11].

Opposed to unipotent subgroups, we have good tori.

DeFINITION 2.13. A good torus is a definable, divisible abelian subgroup in which every definable, connected subgroup is the definable hull of its torsion subgroup.

Introduced in [6] (see also [7]), they proved extremely useful especially since [9]. Similarly to the algebraic dichotomy semi-simple/unipotent, we have the following.

FACT 2.14. [11, Lemma 2.11] Let $H$ be a connected, solvable group of finite Morley rank which is not a good torus. Then there is a non-trivial maximal unipotence parameter $\tilde{p}$ for $H$.

This means that $\tilde{p}$ is a unipotence notion (either finite unipotence of the form $(p, \infty)$, or Burdges' null characteristic unipotence of the form $\left(\infty, d_{\infty}(H)\right)$ with $\left.d_{\infty}(H)>0\right)$ such that $U_{\tilde{p}}(H) \neq 1$, and we now have a unipotent radical to work with.

§3. The analysis. We attack Theorem A.

TheOREM A. Let $G$ be a connected, non-solvable group of finite Morley rank acting definably and faithfully on a connected abelian group $V$ of Morley rank 2 . Then there is an algebraically closed field $\mathbb{K}$ of Morley rank 1 such that $V \simeq \mathbb{K}^{2}$, and $G$ is isomorphic to $\mathrm{GL}_{2}(\mathbb{K})$ or $\mathrm{SL}_{2}(\mathbb{K})$ in its natural action. 
Notation 3.1. Let $G$ be a non-solvable connected group of finite Morley rank acting definably and faithfully on a connected abelian group $V$ of Morley rank 2.

We shall proceed to identifying the action $(G, V)$ with a standard action of $\mathrm{GL}_{2}(\mathbb{K})$ or $\mathrm{SL}_{2}(\mathbb{K})$ on $\mathbb{K}^{2}$. The idea is simple. Our distinction is the following: $\mathrm{GL}_{2}$ has an infinite center, but $\mathrm{SL}_{2}$ hasn't. In each case the center is the intersection of all Borel subgroups. So we need a Borel subgroup, which will by definition contain a connected stabilizer. Reversing the last three sentences, we find the outline of a proof.

In 3.1-3.3 we deal with generalities. We introduce and study a Borel subgroup $B$ in 3.4-3.10. Points 3.11-3.14 are devoted to understanding intersections of $B$; from there on, we construct the center of $G$ and conclude quickly. (Recall that a less general proof can be found in $\S 4$.)

Before we start, here is a remark on solvable subgroups of $G$ admitting more than one minimal subspace.

Lemma 3.2. Let $T \leq G$ be a definable, connected solvable subgroup that normalizes two distinct minimal subgroups of $V$. Then $T$ is a good torus of rank $\leq 2$.

Proof. Assume that $T$ normalizes both $V_{1}$ and $V_{2}$. By faithfulness of the action and Fact 2.3 $T$ is abelian. Let $K=C_{T}\left(V_{1}\right)$ and $L=C_{T}\left(V_{2}\right)$. Then $K \cap L=1$, so by Fact 2.1 both $K^{\circ}$ and $L^{\circ}$ embed into good tori of rank 1 . We may assume that both are proper in $T$.

By Fact $2.1 K$ and $L$ have corank at most 1 in $T$. If they do not generate $T$ then one must have $K^{\circ}=L^{\circ}=1$, so $T$ has rank 1 and $T / K$ is a good torus of rank 1; clearly $T$ is one too.

If $T=K+L$, then $T=K^{\circ}+L^{\circ}$ has rank $\leq 2$ and is easily proved a good torus again.

Recall that an infinite group of finite Morley rank is said to be minimal if it has no proper definable infinite subgroup. If $A$ is such a group, then any definable group of automorphisms of $A$ has rank $\leq \operatorname{rk} A$ [13, Proposition 3.12].

Claim 3.3. $V$ is $G$-minimal but not minimal. It is either torsion-free or an elementary abelian p-group for some prime number $p$.

Proof. $V$ can't be minimal, as otherwise $G$ would have rank $\leq 2$, and therefore be solvable. If $V$ is not $G$-minimal then there is a $G$-invariant $V_{1}<V$ of rank 1 . In this case, $G / C_{G}\left(V_{1}\right)$ acts faithfully on the minimal group $V_{1}$, and must have rank $\leq 1$. Besides, $C_{G}\left(V_{1}\right) / C_{G}\left(V_{1}, V / V_{1}\right)$ acts faithfully on the minimal group $V / V_{1}$, and must have rank $\leq 1$. Now clearly $\left[V, C_{G}\left(V_{1}, V / V_{1}\right), C_{G}\left(V_{1}, V / V_{1}\right)\right]=1$ so $C_{G}\left(V_{1}, V / V_{1}\right)$ is abelian, and $G$ is an extension of rank $\leq 2$ of the latter, hence solvable. This contradiction proves that $V$ is $G$-minimal. 
The second statement now follows from Macintyre's theorem on abelian groups, together with the remark that $V$ can't have divisible torsion by rigidity of $p$-tori [5, Theorem 6.16].

Notation 3.4. Let $v_{0} \in V^{\#}$. Let $H=C_{G}^{\circ}\left(v_{0}\right)<G$.

Clearly $H$ has corank at most 2 in $G$. Our first step is to argue that there is a Borel subgroup of $G$ containing $H$.

Claim 3.5. $H$ is solvable.

Proof. If $V$ is not $H$-minimal, then there is $V_{1}<V$ which is $H$-minimal. Let $K=C_{H}\left(V_{1}\right)$ and $L=C_{K}\left(V / V_{1}\right)$. Notice that $[L,[L, V]]=\left[L, V_{1}\right]=1$ implies that $L$ is abelian. As $K / L$ acts faithfully on the minimal group $V / V_{1}$, it is abelian, so $K^{\circ}$ is solvable. As $H / K$ acts faithfully on the minimal group $V_{1}$, it is abelian, so $H$ is solvable.

So we now suppose that $V$ is $H$-minimal. Then $H$ acts definably and faithfully on the connected abelian group $V$ of rank 2 . As $H<G$, we may apply induction. If $H$ is not solvable, then $H \simeq \mathrm{SL}_{2}(\mathbb{K})$ or $\mathrm{GL}_{2}(\mathbb{K})$, and we find a contradiction to Lemma 2.9.

Notation 3.6. Let $B \geq H$ be a Borel subgroup.

Claim 3.7. $V$ is not B-minimal.

Proof. Otherwise $B$ is abelian by Fact 2.3 , so there is an interpretable field $\mathbb{L}$ such that $V \simeq \mathbb{L}_{+}$and $B$ embeds into $\mathbb{L}^{\times}$. In particular, $H=$ $C_{G}^{\circ}\left(v_{0}\right) \leq B$ must act freely on $V^{\#}$, a contradiction.

Notation 3.8. Let $V_{1}<V$ be a $B$-minimal subgroup.

We want the Borel subgroup $B$ to behave as in $\mathrm{SL}_{2}$ or $\mathrm{GL}_{2}$. The next step is more delicate than the others; we shall prove that $B$ is not morally semi-simple.

Claim 3.9. B is not a good torus.

Proof. Assume it is. We shall show that $G$ has rank 3, find a quotient isomorphic to $\mathrm{PSL}_{2}$, and derive a contradiction.

Step 1: $G$ has rank 3. Let $K=C_{H}\left(V_{1}\right)$ and $L=C_{H}\left(V / V_{1}\right)$. Consider the set $X=K \cup L$. If $X \subsetneq H$, then any $h \in H \backslash X$ has a non-trivial image in $H / K$, which by Fact 2.1 acts freely on $V_{1}^{\#}$, and in $H / L$, which acts freely on $\left(V / V_{1}\right)^{\#}$. As $H=C_{G}^{\circ}\left(v_{0}\right)$, the latter implies $v_{0} \in V_{1}$, and the former a contradiction. It follows that $X=H$, and in particular $H$ is equal to $K$, or to $L$.

Let $U=C_{H}^{\circ}\left(V_{1}, V / V_{1}\right)=(K \cap L)^{\circ}$. We claim that $U=1$. Assume that $V$ has exponent $p$. As $U$ centralizes $V / V_{1}$, for each $u \in U$ and $v \in V$, there is $v_{1} \in V_{1}$ with $v^{u}=v+v_{1}$. Applying $u$ again and again, we end up with $v^{u^{p}}=v+p v_{1}=v$, whence $u^{p} \in C_{G}(v)$, and as this is true regardless of $v$, we find $u^{p}=1$. So $U$ is a $p$-unipotent subgroup of $H \leq B$; by assumption, $U=1$. Let us now deal with the characteristic 0 case. Let $u \in U$ be torsion, say $u^{n}=1$. For any $v \in V$, there is $v_{1} \in V_{1}$ with $v^{u}=v+v_{1}$. Applying $u$ again and again yields this time $v^{u^{n}}=v=v+n v_{1}$, so $n v_{1}=0$ and as $V$ 
is torsion-free, $v_{1}=0$, i.e., $u \in C_{G}(v)$ and $u \in C_{G}(V)$. It follows that the torsion subgroup of $U$ is trivial; as $B$ is a good torus, $U=1$ again.

Fact 2.1 applied to the action of $K^{\circ} /(K \cap L)$ on $V / V_{1}$ and of $L^{\circ} /(K \cap L)$ on $V_{1}$ now shows that $\operatorname{rk} K \leq 1$ and $\operatorname{rk} L \leq 1$. As $H$ is either $K$ or $L$, we deduce $\operatorname{rk} H=1$. Recall that $H$ has corank $\leq 2$ in $G$; hence as $G$ is non-solvable, it has rank 3.

Step 2: finding a subgroup of rank 2. We now find a subgroup of $G$ of rank 2. We may assume that $B$ has rank 1 , so $B=H$ is a good torus of rank 1. In particular it contains a $p$-torus $\tau$ for some prime number $p$, and $B=d(\tau)$. Notice that $p$ can't be the exponent of $V$, as otherwise $V \rtimes \tau$ is a solvable $p$-subgroup of $V \rtimes G$, and general structure results [5, $\S 6.4]$ imply $\tau \leq C(V)=1$, which is absurd. Pick an element $t \in \tau$ of order $p^{n}$. By coprime action [2, Corollary I.9.11], $V=C_{V}(t) \oplus[V, t]$ and both factors are connected. As $v_{0} \in C_{V}(t)$, we deduce that $V_{0}:=C_{V}(t)$ has Morley rank exactly 1 . Now if $s \in \tau$ is a root of $t$ of order $p^{m}>p^{n}$, then the same applies to $s$, and in particular $V_{0}=C_{V}(s)$. Thus $V_{0}=C_{V}(\tau)=C_{V}(B)$.

It follows that if $g \notin N_{G}\left(V_{0}\right)$ and $v \in V_{0} \cap V_{0}^{g}$, then $C_{G}(v) \geq B, B^{g}$. As a conclusion, $V_{0}$ is disjoint from its distinct conjugates, and in particular rk $V_{0}^{G}=\operatorname{rk} G-\operatorname{rk} N_{G}\left(V_{0}\right)+\operatorname{rk} V_{0} \leq 2$ which together with $G$-minimality of $V$ forces rk $N_{G}\left(V_{0}\right)=2$.

Step 3: contradiction. $G / Z(G)$ is a simple group of rank 3 having a definable subgroup of rank 2. By Fact 2.4, $G / Z(G)$ is isomorphic to $\operatorname{PSL}_{2}(\mathbb{K})$ for some interpretable field $\mathbb{K}$. Then no Borel subgroup of $G$ can be a good torus, against the assumption that $B$ is one. This is a contradiction.

Now that we know that $B$ is not a good torus, we reach a more algebraic landscape.

Claim 3.10. $B=N_{G}^{\circ}\left(V_{1}\right)$ and $N_{G}(B)=N_{G}\left(V_{1}\right)$.

Proof. $N_{G}^{\circ}\left(V_{1}\right)$ is easily proved solvable. As $B$ is a Borel subgroup, we have $B=N_{G}^{\circ}\left(V_{1}\right)$. Also, Lemma 3.2 and Claim 3.9 imply that $V_{1}$ is the only $B$-minimal subgroup of $V$. Therefore $N_{G}(B)=N_{G}\left(V_{1}\right)$.

A new tool comes into the picture: unipotence. The reader not satisfied with the minimal account of $\S 2.5$ is directed to [11]. As $B$ is not a good torus, it admits a non-trivial unipotence parameter (Fact 2.14).

Notation 3.11. Let $\tilde{q} \neq(\infty, 0)$ be a maximal unipotence parameter for $B$ and let $U=U_{\tilde{q}}(B)$.

In general, dealing with Burdges' characteristic 0 unipotence requires care. Here the situation is excellent, insofar as only one unipotence degree is possible. The reader may skip the following (which requires absorbing a good deal of null characteristic unipotence) and think of Claims 3.13-3.15 only in the finite characteristic case. For his intellectual satisfaction, the reader would then complete the proof of Theorem A by having a look at the null characteristic argument of Section 4. 
Claim 3.12. If $V$ is torsion-free, then $d_{\infty}(V)=d_{\infty}(G)=1$. Moreover $\tilde{q}=(p, \infty)$ iff $V$ has exponent $p ; \tilde{q}=(\infty, 1)$ iff $V$ is torsion-free. In other words, $U$ and $V_{1}$ have the same unipotence parameter. In particular, $U \leq C_{G}^{\circ}\left(V_{1}\right)$.

Proof. If $V$ is torsion-free, then as $V$ is not minimal by Claim 3.3, $d_{\infty}(V)=1$. For all the remaining statements, consider the solvable group $V U$ in the light of Burdges' structure theorem for nilpotent groups [11, Fact 2.5].

The main tool when using unipotence is Jaligot's Rigidity Lemma which asserts that Borel subgroups tend not to share unipotence. We give here a quite trivial form. The reader may have already met a more subtle version in which some control of the unipotence degree is required; but such control is here provided by Claim 3.12.

A Sylow $\tilde{p}$-subgroup is a definable, connected nilpotent subgroup which is equal to its $U_{\tilde{p}}$-radical, and maximal with respect to these properties.

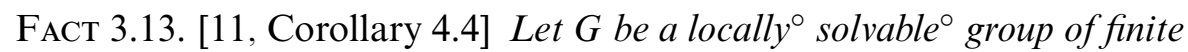
Morley rank, $\tilde{p}=(p, r)$ a unipotence parameter with $r>0$ such that $d_{p}(G)=$ $r$. Let $B$ be a Borel subgroup of $G$ such that $d_{p}(B)=r$. Then $U_{\tilde{p}}(B)$ is a Sylow $\tilde{p}$-subgroup of $G$, and if $U_{1}$ is a non-trivial definable $\tilde{p}$-subgroup of $B$, then $U_{\tilde{p}}(B)$ is the unique Sylow $\tilde{p}$-subgroup of $G$ containing $U_{1}, N\left(U_{1}\right) \leq$ $N\left(U_{\tilde{p}}(B)\right)=N(B)$, and $B$ is the unique Borel subgroup of $G$ containing $U_{1}$.

On the other hand, we have not explained what is local ${ }^{\circ}$ solvability $^{\circ}$, and why $G$ should enjoy such a property. In [11], following Thompson and in spite of another traditional terminology, a group of finite Morley rank $G$ is said to be locally ${ }^{\circ}$ solvable ${ }^{\circ}$ if whenever $1<A \leq G$ is a definable, connected, solvable subgroup, then so is $N_{G}^{\circ}(A)$. The reader will not be surprised to hear that we do not claim that our group $G$ is locally ${ }^{\circ}$ solvable $^{\circ}$ : after all, we are trying to identify $\mathrm{GL}_{2}(\mathbb{K})$ ! But it turns out that in order to prove and use Fact 3.13, the following weaker condition suffices.

Claim 3.14. If $1<U \leq G$ is a nilpotent $\tilde{q}$-unipotent group, then $N_{G}^{\circ}(U)$ is solvable.

Proof. Let $N=N_{G}^{\circ}(U)$. If $N$ is not solvable, then $V$ is $N$-minimal. (This was proved above for $H$, first paragraph of the proof of Claim 3.5.) Moreover, $N$ acts definably and faithfully on $V$.

If $N<G$ we apply induction: $N$ is isomorphic to either $\mathrm{SL}_{2}(\mathbb{K})$ or $\mathrm{GL}_{2}(\mathbb{K})$. As $N$ has an infinite, proper normal subgroup, it cannot be isomorphic to $\mathrm{SL}_{2}(\mathbb{K})$. Furthermore, if $N \simeq \mathrm{GL}_{2}(\mathbb{K})$, then $U$ embeds into $\mathbb{K}^{\times}$where $\mathbb{K}$ has rank 1 , so $U \simeq \mathbb{K}^{\times}$is a good torus, a contradiction to $U=U_{\tilde{q}}(B)$.

If $N=G$, then applying Fact 2.2 we find a $\mathbb{K}$-vector space structure on $V$ such that $V \simeq \mathbb{K}^{2}$ and $G$ embeds into $\mathrm{GL}(V)$, and we argue similarly.

We use our Jaligot Lemma (Fact 3.13) in the next step. 
Claim 3.15. Up to changing $v_{0} \in V^{\#}$, we may suppose that B has corank 1 .

Proof. If $H$ has corank 1 we are done as $H \leq B$. So assume that $H$ has always corank 2. Then for each $v_{0} \in V^{\#}, v_{0}^{G}$ is generic, and in particular $G$ is transitive on $V^{\#}$. If $H<B$ we are done. So suppose $H=B$. Recall from Claim 3.12 that $U \leq C_{G}^{\circ}\left(V_{1}\right)$.

Let $g \notin N_{G}\left(V_{1}\right)$. If there is $v \in\left(V_{1} \cap V_{1}^{g}\right)^{\#}$, then $C_{G}^{\circ}(v) \geq U$, $U^{g}$. As $G$ is transitive on $V^{\#}, v$ is conjugate to $v_{0}$, and $C_{G}^{\circ}(v)$ is solvable by Claim 3.5. Now Fact 3.13 (valid here in view of Claim 3.14) forces $g \in N_{G}(B)=$ $N_{G}\left(V_{1}\right)$ by Claim 3.10, a contradiction.

Therefore the subgroups $\left\{V_{1}^{g}\right\}$ are pairwise disjoint, and $N_{G}^{\circ}\left(V_{1}\right)$ must have corank 1. But $N_{G}^{\circ}\left(V_{1}\right)=B$ by Claim 3.10.

We now are ready to recognize the action.

Notation 3.16. Let $N=\bigcap_{g \in G} B^{g}$.

Claim 3.17. If $N$ is infinite, then there is an interpretable field $\mathbb{K}$ of rank 1 such that $V \simeq \mathbb{K}^{2}$ and $G \simeq \mathrm{GL}(V)$.

Proof. Let $g \notin N_{G}(B)=N_{G}\left(V_{1}\right)<G$. By Lemma 3.2, $B \cap B^{g}$ is a good torus; hence $N^{\circ} \neq 1$ is abelian. Then by Fact 2.2, $G$ embeds into $\mathrm{GL}(V)$ for some $\mathbb{K}$-vector space structure on $V$. Clearly rk $\mathbb{K}=1$ and $V \simeq \mathbb{K}^{2}$, so rk $\mathrm{GL}(V)=4$. As $G$ is non-solvable but has an infinite, normal subgroup, it must have rank 4. Therefore $G \simeq \mathrm{GL}(V)$.

CLAIM 3.18. If $N$ is finite, then there is an interpretable field $\mathbb{K}$ of rank 1 such that $V \simeq \mathbb{K}^{2}$ and $G \simeq \operatorname{SL}(V)$.

Proof. $G / N$ is a finite, central quotient of $G$ which contains $B / N$ of corank 1. Fact 2.4 yields $G / N \simeq \mathrm{PSL}_{2}(\mathbb{K})$ for some interpretable field $\mathbb{K}$ of rank 1 . As rk $G=3$ and $G$ is not solvable, one must have $G^{\prime}=G$, that is $G$ is perfect: and therefore a perfect central extension of a quasi-simple group. It is known that $G$ itself is algebraic [2, Proposition 3.1 p. 136]. It follows $G \simeq \mathrm{PSL}_{2}$ or $\mathrm{SL}_{2}$ (arguing for example that $G$ has Lie rank 1). Now Facts 2.5 and 2.7 give the conclusion.

$\S 4$. A shorter proof in characteristic 0 . In this section we prove Theorem A in the special case where $V$ is torsion-free. As definable hulls of elements of $V^{\#}$ are infinite, we will easily find a bound on the rank of $G$. We insist that one cannot provide such a shortcut in characteristic $p$, and this is why we used as our main proof the characteristic-independent argument given in $\S 3$.

THEOREM A IN CHARACTERISTIC 0 . Let $G$ be a connected, non-solvable group of finite Morley rank acting definably and faithfully on a torsion-free connected abelian group $V$ of Morley rank 2. Then there is an algebraically closed field $\mathbb{K}$ of Morley rank 1 and characteristic 0 such that $V \simeq \mathbb{K}^{2}$, and $G$ is isomorphic to $\mathrm{GL}_{2}(\mathbb{K})$ or $\mathrm{SL}_{2}(\mathbb{K})$ in its natural action.

Claim 4.1. rk $G \leq 4$; $G$ has an involution. 
Proof. By Claim $3.3 V$ is $G$-minimal but not minimal. Let $v \in V^{\#}$ and $V_{1}=d(v)$, so that $C(v)=C\left(V_{1}\right)$. Of course $V_{1}<V$ : otherwise $C(v)=1$ and $G$ has Morley rank 2 , hence is solvable. So $V_{1}<V$, and since $V$ has no torsion, $V_{1}$ is a connected group of Morley rank 1.

By $G$-minimality of $V$, there is $g \notin N\left(V_{1}\right)$; clearly $V=V_{1}+V_{1}^{g}$, hence $1=C_{G}(V)=C_{G}\left(V_{1}, V_{1}^{g}\right)=C_{G}\left(v, v^{g}\right)$. Consider the multiplication map $C_{G}(v) \times C_{G}\left(v^{g}\right) \rightarrow G$; we get $2 \operatorname{rk}\left(C_{G}(v)\right) \leq \operatorname{rk} G$, that is $\operatorname{rk} G \leq 2 \operatorname{rk} v^{G} \leq 4$.

More precisely, since rk $G>2$, one also has that $v^{G}$ is generic in $V$. So there is only one non-trivial orbit. In particular, $(-v)^{G}=v^{G}$, and there is $g \in G$ such that $v^{g}=-v$. This gives an element not in $C_{G}(v)$ whose square lies in $C_{G}(v)$; lifting torsion, we get an involution in $G$.

Non-solvability forces the Morley rank of $G$ to be either 3 or 4 .

Claim 4.2. If $\mathrm{rk} G=3$, then there is an interpretable field $\mathbb{K}$ of rank 1 such that $V \simeq \mathbb{K}^{2}$ and $G \simeq \mathrm{SL}(V)$.

Proof. By non-solvability, $G$ must be quasi-simple. If it has no subgroup of corank 1, then it is a quasi-simple bad group, and $G / Z(G)$ is a simple bad group. It is then known that $G / Z(G)$ has no involutions [5, Theorem 13.3]. In particular the finite, connected extension $G$ can have no involutions either by [3]. This contradicts Claim 4.1.

Hence $G$ has a subgroup of corank 1 , and so does $G / Z(G)$. In particular, Fact 2.4 implies that $G / Z(G) \simeq \mathrm{PSL}_{2}(\mathbb{K})$. One then argues as in the end of the proof of Claim 3.18 to conclude that $G \simeq \mathrm{SL}(V)$.

So we may assume that rk $G=4$.

Claim 4.3. G has a definable subgroup of corank 1.

Proof. Let $i$ be an involution of $G$. Notice that $G$ has no non-trivial 2-unipotent subgroups, as they would act trivially on $V$. So making use of [3], the Sylow 2-subgroups of $G$ are toral-by-finite (" $G$ has odd type", following the standard terminology). By Burdges' and Cherlin's torality principle, [8, Theorem 3], there is a Sylow 2-subgroup $S$ of $G$ such that $i \in S^{\circ}$. Let $T=d\left(S^{\circ}\right)$. We shall find a non-trivial $T$-invariant subgroup $V_{1}<V$ inverted by $i$.

If $i$ is central in $G$, then $C_{V}(i)<V$ is $G$-invariant, hence trivial, and $i$ inverts $V$. If $V$ is $T$-minimal, then by Fact 2.1 it is isomorphic to $\mathbb{K}_{+}$ for some algebraically closed field $\mathbb{K}$ of characteristic 0 , hence minimal by [13, Corollaire 3.3]. This contradicts the non-minimality of $V$. Hence $V$ is not $T$-minimal, and $T$ normalizes some $V_{1}<V$, which is necessarily inverted by $i$.

If $i$ is not central in $G$, let $V_{1}=[V, i]>1$. We claim that $V_{1}<V$ : otherwise, for any $g \in G, v \in V$, we find $v^{i g i}=(-v)^{g i}=-\left(v^{g}\right)^{i}=v^{g}$, so $[i, g] \in C_{G}(V)=1$, and $i$ is central in $G$, a contradiction. Here again, $T$ normalizes some $V_{1}<V$ inverted by $i$. 
In either case we find $i \in T \leq N^{\circ}\left(V_{1}\right)$ but $i \notin C\left(V_{1}\right)$, so $N^{\circ}\left(V_{1}\right)>$ $C^{\circ}\left(V_{1}\right)$. As in Claim 4.1, $C^{\circ}\left(V_{1}\right)$ has rank 2, and $N^{\circ}\left(V_{1}\right)<G$, so $N^{\circ}\left(V_{1}\right)$ has Morley rank 3 and we are done.

Proof of Theorem A in Characteristic 0. It suffices to find a vector space structure such that $G \simeq \mathrm{GL}(V)$. Recall from Claims 4.1 and 4.3 that $G$ has Morley rank 4 and a definable subgroup of corank 1. If $G$ is quasi-simple, then $G / Z(G)$ is simple and has a subgroup of corank 1; by Fact $2.4 \operatorname{rk}(G / Z(G))=3$, a contradiction. Hence $G$ has an infinite proper definable normal subgroup $H$.

If $H$ is not solvable, then it has Morley rank 3, and by Claim 4.2 $H \simeq$ $\mathrm{SL}_{2}(\mathbb{K})$. But there are no definable groups of automorphisms of a field of finite Morley rank [5, Theorem 8.3]. Using general results on definable automorphisms of algebraic groups [5, Theorem 8.4], we deduce that $A=$ $C_{G}^{\circ}(H) \triangleleft G$ is infinite. As $A \cap H \leq Z(H)$ is finite, $A$ has Morley rank 1 . In particular it is abelian. So there is an infinite definable abelian normal subgroup. This is of course also the case if $H$ is solvable.

So we may assume that there is an infinite, abelian, definable, normal subgroup of $G$, and one argues as in Claim 3.17 to find $G \simeq \mathrm{GL}(V)$ for some interpretable vector space structure $V \simeq \mathbb{K}^{2}$.

§5. The natural module of $\mathrm{SL}_{2}$. Recall that in order to prove Theorem A we needed only a baby identification result on the natural $\mathrm{SL}_{2}$-module, namely Fact 2.7. In this section we generalize Fact 2.8 to characteristic not 2. Our result is the following.

THEOREM B. Let $\mathbb{K}$ be a field of Morley rank $k$. Let $G$ be a group of finite Morley rank isomorphic to $\mathrm{SL}_{2}(\mathbb{K})$ acting definably and non-trivially on a connected abelian group $V$ of rank $\leq 2 k$. Then $V$ is a natural module for $G$.

If $\mathbb{K}$ has characteristic 2, this is Fact 2.8 (recall that connectednes was implicit in the original statement [2, Lemma II.5.31]). Otherwise we copy its proof by Altınel, Borovik and Cherlin with minor changes. However we now need the original version of Fact 2.6, which also takes $\mathrm{PSL}_{2}$ into account: as in characteristic not $2 \mathrm{SL}_{2}$ is no longer simple, we need to rule out finite kernels.

In this section, "unipotent" bears its standard algebraic meaning.

FACT 5.1. [16, Theorem 3.4] Let $\mathbb{K}$ be a field and let $G \simeq \mathrm{PSL}_{2}(\mathbb{K})$ or $\mathrm{SL}_{2}(\mathbb{K})$. Let $V$ be a $G$-module. Suppose the following:

1. $C_{V}(G)=0$,

2. $V$ is faithful and $[U, U, V]=1$, where $U$ is a maximal unipotent subgroup of $G$.

Let $0 \neq v \in C_{V}(A)$ and $W=\left\langle v^{G}\right\rangle$. Then there exists a field action of $\mathbb{K}$ on $W$ such that $W$ is the natural $G$-module. In particular $G \simeq \mathrm{SL}_{2}(\mathbb{K})$. 
(Bear in mind, when reading [16], that Timmesfeld defines quadracity only for faithful modules.)

Notice that if $v \in C_{V}(A)^{\#}$ is such that $[v, G]=V$, which is typically the case if $V$ is $G$-minimal, then one will have $\left\langle v^{G}\right\rangle=V$. In our proof of Theorem B we shall work with $G$-minimal submodules, which appear slightly more natural (informally speaking) than irreducible ones in the finite Morley rank category. But this captures essentially the same methods.

We actually need two more results before the argument starts.

FACT 5.2 (special case of [14, Théorème 4]). Let $\mathbb{K}$ be a field of Morley rank. Then all definable proper connected subgroups of $\mathrm{SL}_{2}(\mathbb{K})$ are solvable.

FACT 5.3. Let $\mathbb{K}$ be a field of Morley rank and let $H<\mathrm{SL}_{2}(\mathbb{K})$ be a definable subgroup with at least three elements. Then $H$ is contained in at most two Borel subgroups of $\mathrm{SL}_{2}$.

Proof. If $H$ contains a unipotent element it is contained in a unique Borel subgroup. Otherwise $H$ is semi-simple, and if $\beta$ is a Borel subgroup containing $H$ there is an algebraic torus $\tau$ of $\beta$ containing $H$, and $\tau=$ $C_{G}^{\circ}(H) \leq \beta$. In particular the question reduces to the case where $H$ itself is an algebraic torus, for which it is well-known.

Proof of Theorem B. We shall of course use Fact 5.1. As we have made no assumption on faithfulness of the module, perhaps a non-trivial kernel will appear, yielding an action of $\mathrm{PSL}_{2}(\mathbb{K})$, which we want to exclude. So we shall really use the original statement Fact 5.1 instead of its special case Fact 2.6. In particular we need all our following claims to be $\mathrm{PSL}_{2}$ compatible.

NotATiOn 5.4. Let $\mathbb{K} \models \mathrm{ACF}_{\neq 2}$ have Morley rank $k$ and $G \simeq \mathrm{PSL}_{2}(\mathbb{K})$ or $\mathrm{SL}_{2}(\mathbb{K})$. Let $U$ be a maximal unipotent subgroup of $G$ and $B$ its normalizer. We also let $T$ be a maximal torus of $B$, and $\zeta$ a 2-element inverting $T$.

Notice that the order of $\zeta$ depends on the isomorphism type of $G$.

Notation 5.5. Let $V$ denote a connected, abelian group which is a definable, non-trivial $G$-module of rank $\leq 2 k$.

We shall prove that $V$ is a natural module for $G$ (this will show in particular rk $V=2 k$ and $\left.G \simeq \mathrm{SL}_{2}(\mathbb{K})\right)$.

Claim 5.6. We may assume that $V$ is $G$-minimal.

Proof. Assume Theorem B holds in the $G$-minimal case, and let $V$ be as in Notation 5.5. If $W<V$ is $G$-minimal, then $W$ or $V / W$ is not trivial under $G$ : otherwise $[[V, G], G]=1$ forces $\left[V, G^{\prime}\right]=1$, but regardless of the isomorphism type of $G$ one has $G^{\prime}=G$, so $V$ is $G$-trivial, a contradiction.

As by assumption all non-trivial $G$-minimal sections have rank $2 k$, it follows that any non-trivial module of rank $\leq 2 k$ has rank exactly $2 k$ and it $G$-minimal. 
Claim 5.7. We may assume that $C_{V}(G)=0$.

Proof. Assume Theorem B holds when the (right-)kernel is trivial. As we may assume that $V$ is $G$-minimal by Claim 5.6, $C_{V}(G)$ is finite. Let $W=V / C_{V}(G) ; W$ is $G$-minimal. If $x \in V$ is such that $\bar{x} \in W$ lies in $C_{W}(G)$, then $[G, x]$ is a connected subgroup of $C_{V}(G)$ which is finite, hence $[x, G]=0$. It follows $x \in C_{W}(G)$ and $\bar{x}=0$, that is $C_{W}(G)=0$.

By assumption, Theorem $\mathrm{B}$ applies to $W$, which is therefore a natural module for $G \simeq \mathrm{SL}_{2}(\mathbb{K})$. We then proceed exactly as in the second paragraph of the proof of Fact 2.7 to show $C_{V}(G)=0$, that is $W=V$.

From now on $V$ is $G$-minimal and $C_{V}(G)=0$. We shall identify $V$ with the natural module for $G$.

Notation 5.8. Let $V_{1} \leq V$ be a $B$-minimal subgroup.

Notice that by Fact 2.3, $U$ centralizes $V_{1}$. In particular $V_{1}<V$ as otherwise $U$ centralizes $V$, but conjugates of $U$ generate $G$, so the action is trivial.

Notation 5.9. For $v \in V_{1}$, let $q(v)=v+v^{\zeta}$.

The function $q$ is a definable group homomorphism from $V_{1}$ to $V$ which will play a crucial role in the following key properties.

Claim 5.10. rk $V=2 k$; moreover $V_{1}=C_{V}^{\circ}(U)$ has rank $k$ and is disjoint from its distinct conjugates.

Proof. Step 1: a disjoint subgroup. Let $\hat{V}_{1}=C_{V}^{\circ}(U)$. Recall that $U$ centralizes $V_{1}$, so $V_{1} \leq \hat{V}_{1}$. Now notice that $N_{G}^{\circ}\left(\hat{V}_{1}\right)=B$. Furthermore, if $g \notin B$ then $G=\left\langle U, U^{g}\right\rangle$ centralizes $\hat{V}_{1} \cap \hat{V}_{1}^{g}$, which implies $\hat{V}_{1} \cap \hat{V}_{1}^{g}=0$ as $C_{V}(G)=0$. In other words $\hat{V}_{1}$ is disjoint from its distinct conjugates, and a trivial rank computation yields

$$
\operatorname{rk} \hat{V}_{1}^{G}=\operatorname{rk} \hat{V}_{1}+\operatorname{rk} G-\operatorname{rk} N_{G}\left(\hat{V}_{1}\right) \leq \operatorname{rk} V .
$$

In particular rk $V_{1} \leq \mathrm{rk} \hat{V}_{1} \leq k$.

If rk $V_{1} \geq k$, then $V_{1}=\hat{V}_{1}$ is disjoint from its distinct conjugates, and this certainly forces $\mathrm{rk} V=2 k$. So assume rk $V_{1}<k$. We shall reach a contradiction using the morphism $q$ (Notation 5.9).

Step 2: Many elements share a big centralizer. The idea is to have a common semi-simple subgroup $T_{1} \leq T$ centralize infinitely many elements of $V_{1}$ - to make sure that we do use the same element $\zeta$ when computing values of the morphism $q$.

Let $O=v_{0}^{T}$ be a $T$-orbit of maximal rank in $V_{1}$ and let $T_{1}=C_{T}^{\circ}(O)$. As $T$ is abelian, $T_{1}=C_{T}^{\circ}\left(v_{0}\right)$. Now $U \leq C_{B}^{\circ}\left(v_{0}\right)$ so $C_{G}^{\circ}\left(v_{0}\right)=C_{B}^{\circ}\left(v_{0}\right)=U \rtimes T_{1}$ and our assumption on $\operatorname{rk} V_{1}$ yields $\operatorname{rk} v_{0}^{B}<k$, so rk $T_{1} \neq 0$. Hence $T_{1}$ is non-trivial.

Let $V_{2}=C_{V}^{\circ}\left(T_{1}\right)$. If $O$ is finite, then by connectedness and maximality of rk $O, T$ centralizes $V_{1}$, so $V_{2} \geq V_{1}$. Otherwise $d^{\circ}(O) \leq V_{2}$, proving that 
$V_{2}$ is infinite in any case. Notice that for all $v \in V_{2}^{\#}, C_{G}^{\circ}(v)$ contains $U \rtimes T_{1}$ (i.e., $T_{1}$ is common to all $v$ in an infinite subgroup $V_{2}$ of $V_{1}$ ).

Step 3: For all $v \in V_{2}^{\#}, C_{G}^{\circ}(q(v))=T$. Consider the element $q(v)$ for $v \in V_{2}^{\#}$. If $q(v)=0$, then $\zeta$ inverts $v$, so $U^{\zeta}$ centralizes $v$. Hence $C_{G}^{\circ}(v) \geq$ $U, U^{\zeta}$, whence $C_{G}^{\circ}(v)=G$ by Fact 5.2. This contradicts $C_{V}(G)=0$. Therefore $q$ is injective.

Still for $v \in V_{2}^{\#}$, consider $H=C_{G}^{\circ}(v)<G$ and $K=C_{G}^{\circ}(q(v))<G$. By construction, $T_{1} \leq K$, so by Facts 5.2 and 5.3, only $B$ and $B^{\zeta}$ can possibly contain $K$. Assume only, say $B$, does. This implies that $U \cap K \neq 1$, so there is $1 \neq x \in U \cap K$. Then $x$ centralizes $v$ and $q(v)$, so it centralizes $v^{\zeta}$. Now regardless of the isomorphism type of $G, x^{\zeta}=x^{\zeta^{-1}}$ centralizes $v$. Hence $H$ meets both $U$ and $U^{\zeta}$, a contradiction. We show $K \leq B$ similarly. Thus $K \leq B \cap B^{\zeta}$, which shows rk $K \leq k$. Hence $\operatorname{rk} q(v)^{G} \geq 2 k$.

As rk $V \leq 2 k$, this implies that $C_{G}^{\circ}(q(v))=T$ and $q(v)^{G}$ is generic in $V$.

Step 4: Contradiction. Let $v^{\prime} \in V_{2}^{\#}$. Then by genericity of $q\left(v^{\prime}\right)^{G}$ in $V$ there is $g \in G$ such that $q\left(v^{\prime}\right)=q(v)^{g}$. Therefore $g$ normalizes $T=C_{G}^{\circ}(q(v))=C_{G}^{\circ}\left(q\left(v^{\prime}\right)\right)$, and $g \in N(T)=T \cdot\langle\zeta\rangle$. So $q\left(v^{\prime}\right) \in$ $\left\{q(v), q(v)^{\zeta}, q(v)^{\zeta^{2}}, q(v)^{\zeta^{3}}\right\}$ (this set might be extremely redundant). Hence the morphism $q$ takes finitely many values, a contradiction as it was proved injective and $V_{2}$ is infinite.

Therefore $V_{1}=\hat{V}_{1}$; as explained why in Step 1, everything is proved.

After the previous technical result, the analysis is quickly at an end.

Claim 5.11. $V=V_{1} \oplus V_{1}^{\zeta}$, and $T$ acts transitively on $V_{1}^{\#}$ and $\left(V_{1}^{\zeta}\right)^{\#}$.

Proof. By Claim 5.10 $V_{1}$ is disjoint from its non-trivial conjugates. As $B \leq N_{G}\left(V_{1}\right)<G$, by Fact $5.2 N_{G}\left(V_{1}\right)$ is a finite extension of $B$, which is self-normalizing; hence $B=N_{G}\left(V_{1}\right)$. In particular $V_{1}^{\zeta} \neq V_{1}$. It follows from Claim 5.10 that $V=V_{1} \oplus V_{1}^{\zeta}$.

Notice that $V_{1}$ is $T$-minimal. Let $t \in C_{T}\left(V_{1}\right)$. Then $t^{\zeta}$ centralizes $V_{1}^{\zeta}$, so $t$ centralizes $V_{1}+V_{1}^{\zeta}=V$, that is $t \in C_{G}(V)$. We have made no assumption on faithfulness, but whether $G$ be $\mathrm{PSL}_{2}(\mathbb{K})$ or $\mathrm{SL}_{2}(\mathbb{K}), C_{G}(V)$ must be finite. Hence $C_{T}\left(V_{1}\right)$ is finite, and $\mathrm{rk} T / C_{T}\left(V_{1}\right)=k$.

It follows from Fact 2.1 that $T$ is transitive on $V_{1}^{\#}$. The same is true on $\left(V_{1}^{\zeta}\right)^{\#}$.

Claim 5.12. $[V, U, U]=0$.

By Claim 5.11, $V=V_{1} \oplus V_{1}^{\zeta}$ and $T$ acts transitively on $V_{1}^{\#}$ and on $\left(V_{1}^{\zeta}\right)^{\#}$. So $T$ acts transitively on $\left(V / V_{1}\right)^{\#}$, and $C_{V / V_{1}}(U)$ is either 0 or $V / V_{1}$. We shall prove it is $V / V_{1}$.

We turn our attention to the solvable group $V / V_{1} \rtimes B$. The quotient $\left(V / V_{1} \rtimes B\right) / F^{\circ}\left(V / V_{1} \rtimes B\right)$ is abelian by a classical property of connected, solvable groups of finite Morley rank [5, Theorem 9.21.]. Clearly $\left[V / V_{1}, T\right]=$ 
$V / V_{1}$, so $T$ has a non-trivial image modulo $F^{\circ}\left(V / V_{1} \rtimes B\right)$. Furthermore, $[T, U]=U$; it follows $U \leq F^{\circ}\left(V / V_{1} \rtimes B\right)$. In other words, $K=V / V_{1} \rtimes U$ is nilpotent. In particular $Z(K) \cap V / V_{1} \neq 0$, i.e., $C_{V / V_{1}}(U) \neq 0$. This shows that $U$ centralizes $V / V_{1}$. Hence $[V, U] \leq V_{1}=C_{V}^{\circ}(U)$ and $[V, U, U]=0$.

We now finish the proof of Theorem B.

In view of Claim 5.12 all assumptions of Fact 5.1 are satisfied, except perhaps faithfulness. If $G$ is not faithful on $V$, then $G \simeq \mathrm{SL}_{2}(\mathbb{K})$ and the kernel of the action is $Z(G)$. Then $G / Z(G) \simeq \mathrm{PSL}_{2}(\mathbb{K})$ is faithful on $V$; by Fact $5.1 G / Z(G) \simeq \mathrm{SL}_{2}(\mathbb{K})$, a contradiction.

So $G$ is faithful on $V$, and by Fact 5.1 again $V$ is a natural module for $G \simeq \mathrm{SL}_{2}(\mathbb{K})$.

\section{REFERENCES}

[1] Tuna Altinel, Alexandre Borovik, and Gregory Cherlin, Groups of mixed type, Journal of Algebra, vol. 192 (1997), no. 2, pp. 524-571.

[2] - Simple groups of finite Morley Rank, American Mathematical Society, 2008.

[3] Alexandre BoroviK, JefFrey Burdges, and Gregory Cherlin, Involutions in groups of finite Morley rank of degenerate type, Selecta Mathematica. New Series, vol. 13 (2007), no. 1, pp. 1-22.

[4] Alexandre Borovik and Gregory Cherlin, Permutation groups of finite Morley rank, Model theory and applications to algebra and analysis, Cambridge University Press, 2005.

[5] Alexandre BoroviK and Ali Nesin, Groups of finite Morley rank, Oxford Logic Guides, vol. 26, The Clarendon Press Oxford University Press, New York, 1994, Oxford Science Publications.

[6] JEFFREY BURDGES, A signalizer functor theorem for groups of finite Morley rank, Journal of Algebra, vol. 274 (2004), pp. 215-229.

[7] - Simple groups of finite Morley rank of odd and degenerate type, $\mathrm{Ph} . \mathrm{D}$. thesis, Rutgers University, New Brunswick, New Jersey, 2004.

[8] JefFrey Burdges and Gregory Cherlin, Semisimple torsion in groups of finite Morley rank, submitted, 2008.

[9] Gregory ChERLIn, Good tori in groups of finite Morley rank, Journal of Group Theory, vol. 8 (2005), no. 5, pp. 613-621.

[10] Adrien Deloro, Groupes simples connexes minimaux algébriques de type impair, Journal of Algebra, vol. 317 (2007), no. 2, pp. 877-923.

[11] Adrien Deloro and Eric Jaligot, Groups of finite Morley rank with solvable local subgroups, available at arXiv:0802.1394v2 [math.GR], submitted, 2007.

[12] Dugald Macpherson and Anand Pillay, Primitive permutation groups of finite Morley rank, Proceedings of the London Mathematical Society. Third Series, vol. 70 (1995), no. 3, pp. 481-504.

[13] Bruno Poizat, Groupes stables, Nur al-Mantiq wal-Ma'rifah, Villeurbanne, 1987.

[14] — Quelques modestes remarques à propos d'une conséquence inattendue d'un résultat surprenant de Monsieur Frank Olaf Wagner, The Journal of Symbolic Logic, vol. 66 (2001), pp. 1637-1646.

[15] Vladimir L. Popov, Generically multiple transitive algebraic group actions, Algebraic groups and homogeneous spaces, Tata Institute of Fundamental Research Studies in Mathematics, Tata Institute of Fundamental Research, Mumbai, 2007, pp. 481-523. 
[16] Franz Georg TimmeSFeld, Abstract root subgroups and simple groups of Lie-type, Monographs in Mathematics, vol. 95, Birkhäuser, Basel, 2001.

DEPARTMENT OF MATHEMATICS, RUTGERS UNIVERSITY

HILL CENTER, BUSCH CAMPUS

110 FRELINGHUYSEN ROAD, PISCATAWAY, NJ 08854, USA

E-mail: adeloro@math.rutgers.edu 